\title{
MONOPOLY AS A MARKOV PROCESS
}

\author{
ROBERT B. ASH AND RICHARD L. BISHOP
}

\begin{abstract}
The limit frequencies of the positions in the game of Monopoly are calculated on the basis of Markov chains. In order to make the process Markovian some minor modifications in the rules are necessary. A parameter is introduced so that by varying this parameter we can determine how distorted our mode is compared with the actual game. The convergence properties of Markov chains are so nice that it was feasible to use hand computation. A neat trick with power series played an important role in determining how the limit frequencies depend on the parameter. A method for determining higher eigenvalues is illustrated. These eigenvalues yield a good estimate on the rapidity of convergence to the limit frequencies: to within one percent after 20 turns. Finally, expected income from the bank and expected rents on properties are calculated from the limit frequencies. This allows us to give a quantitative estimate of the relative value of the various properties.
\end{abstract}

\section{THE PROBLEM}

Practically everyone is familiar with the rudiments, anyway, of the game of Monopoly. Pawns are marched around a board with 40 positions according to the dictates of a pair of dice. The person in control of the pawn pays out or receives money to or from other players and the bank, depending on the position of the pawn and the state of development of the particular position. Thus, In order to assess the relative value of controlling the various properties one must have an idea of the frequencies with which it can be expected that pawns will occupy each position. With a knowledge of such frequencies, one should also be able to calculate the expected income from the bank, and thus determine whether or not bankruptcy is likely for a given distribution of property. Of course, those who play Monopoly know that short-term accidents often have a decisive effect. However, lacking any control over these short-term accidents, the best strategy would seem to be to base decisions on the limit frequencies. This should at least give favorable results over a large number of games.

\section{MARKOV PROCESSES}

A discrete time, finite state Markov process (also called a finite Markov chain) is a system having a finite number of attitudes or states, which proceeds sequentially from one state to another, and for which the probability of passing from state $i$ to state $j$ is a number $p_{i j}$ which depends only on $i$ and $j$, and not, say, on the history of the system in attaining state $i$. We shall only be concerned here with the situation in which $p_{i j}$ does not depend on the time when the transition from $i$ to $j$ is made. In this case, the chain is said to have stationary transition probabilities. For more details see [F, Chapters 15, 16]. 
In Monopoly, the basic quantity of interest for strategic purposes is the probability of being on any of the 40 positions in a given turn. It seems natural to use the turn as the unit of time and the position at the start of the turn as the state. However, this process fails to be Markovian. Indeed, if a pawn is in the jail state, the person in control sometimes may choose whether to stay in jail if doubles are not thrown. The allowability of this choice does depend on the history of the previous two states: if a pawn has been in jail two turns it must be moved out of jail.

Another feature of the game which makes the process non-Markovian is the chance and community chest position-transfer cards. There are three positions each called chance and community chest, and upon reaching such a position the top card of 16 chance cards or 16 community chest cards is drawn. Of these cards, 10 chance cards and 2 community chest cars require a movement to another position. The drawn card is placed at the bottom of the stack after following its instructions, except for one "get out of jail free" card in each stack, which may be kept until used. Thus, the order in which these cards appear, and hence their effect, does not depend upon the position of the pawn, but upon the initial order in the stack and the history of their usage.

\section{Simplifying ASSUMPTIONS}

We can make the process for Monopoly Markovian by (a) distinguishing three, rather than one, jail states and (b) changing the rules slightly so as to remove the choice of the player in leaving jail and the historical dependence on the order of the chance and community chest cards.

(a): We shall consider the jail position as determining three states: in jail, having just arrived on the previous turn; in jail, having stayed one turn; and in jail, having stayed two turns.

(b): We deprive the player of his choice in leaving jail. We want to allow some flexibility in strategy here - in the initial game players are interested in accumulating property and hence in minimizing time in jail; in the end game players are interested in avoiding high rents and hence in maximizing time in jail. Thus, we insert a parameter $p$, which is defined to be the probability that the player will decide to leave jail when that choice is available. Of course, it only applies to the 5/6 of turns when doubles are not thrown; when doubles are thrown the pawn must move out of jail.

We shall obtain verification of the generally accepted strategies, and even a measure of their effectiveness: $p=1$ in the beginning, $p=0$ in the end. The expected frequencies will be evaluated as a function of $p, 0 \leq p \leq 1$. Notice that when $p=1$ two of the jail states cannot be reached.

The change in procedure for leaving jail could be attained by using a random number generator to make the choice. We can use another randomizing device to eliminate the historical feature from the card draws: instead of replacing the card at the bottom of the deck, the whole deck will be shuffled after each draw. So as to maintain equal probabilities, the "get out of jail free" cards are also replaced immediately, with a system of credit replacing the physical use of these cards. Thus, on any draw we shall assume that any one of the cards of that pile will be turned with probability $1 / 16$. 
When a position-transfer card is drawn, we do not consider the pawn to have been in the state of the chance or community chest position for that turn, but we count only the state to which the transfer is made. Thus, the chance positions will have a listed frequency which is $3 / 8$ the actual frequency of lighting on the position, and the community chest positions will have a listed frequency which is $7 / 8$ the actual frequency of the position. Of course, this convention will make no difference in rent calculations The advantage is that it makes the sum of the the frequencies of states equal to 1 .

Having made these changes in the rules, we obtain a model of the motion of a pawn around a Monopoly board as a Markov chain having 42 states (the "Go to jail" position is not considered to be a state). We are confident that these assumptions do not vitiate our results, particularly since our goal is the limit frequencies, representing an average over a great number of turns.

\section{Position fRequencies}

For each of the 42 states there is an associated position; we shall also be able to assign limit frequencies to each of these states. However, during each turn the pawn may light upon up to three positions, due the rule on doubles throws. Thus, although the sum of the limit frequencies of the 42 states is unity, the sum of the limit frequencies of lighting on the 40 positions is somewhat greater. It is these limit frequencies for positions, the position frequencies per turn, in which we are primarily interested, since they determine expected income and rent per turn.

We can estimate how much greater than 1 the sum of the position frequencies is, provided we neglect the possibility of losing an extra throw after a throw of doubles due to going "directly to jail". The rules provide for such incarceration, with loss of the extra throw, upon drawing a "go to jail" card or upon lighting on a certain board position. Aside from this, on a given turn, with probability $5 / 6$, doubles are not thrown and only one position on the board is occupied; with probability $(1 / 6)(5 / 6)$, doubles are thrown exactly once and two position are occupied; with probability $1 / 36$, doubles are thrown twice and three position are occupied. On this basis the average number of positions occupied per turn would be

$$
(5 / 6) \cdot 1+(5 / 36) \cdot 2+(1 / 36) \cdot 3=43 / 36 .
$$

The same feature, allowing another throw when doubles are thrown, complicates the calculation for the transition frequencies. for example, the determination of the probability of advancing 11 spaces in a complete turn must include the following:

- $1 / 18$, the probability of throwing 11 ,

- $1 / 81$, the probability of throwing one doubles and then another throw to total 11 ,

- $(1 / 36)(1 / 36)(5 / 6)$, the probability of two doubles and another throw making 11 ,

- the probability of advancing 11 by other means using position transfers.

In particular, the transition matrix $\left(p_{i j}\right)$ has very few entries which are 0 , but is filled up with tiny numbers which are difficult to calculate and keep track of. This would not bother a modern computer, but for the computations to be done by hand as one of us (RLB) did, it is desirable to look for a better way of organizing the problem. Such an approach is discussed in the next section. 


\section{Another Markov chain}

We can reduce the complication caused by multiple positions during a turn by changing the basic unit of time from a turn to a throw (of the dice). Then, in order to avoid having the transition probabilities depend on previous states, we increase the number of states, so that a state will include not only a position of the pawn, but also the number of the throw within a turn. This does not apply to the jail position since it is impossible to be in jail and simultaneously be about to take the second or third throw of a turn. Thus, besides the three jail states, which we number 300, 301, 302, the new Markov chain has 117 states. We call the new chain the per throw chain as opposed to the per turn chain of $\S 3$. We number the states as follows, where the integer $n$ is the number of steps ahead from Go:

- state $n$ : on position $n$ at the beginning of a turn

- state $n^{\prime}$ : on position $n$, to throw dice the second time, having thrown doubles the first throw of the turn

- state $n^{\prime \prime}$ : on position $n$, to throw dice the third time, having thrown doubles the first two throws of the turn.

Hence $n$ runs from $0(=$ Go) through 39 (= Boardwalk), except for the number $30(=$ Go to jail, which is impossible to remain on).

As a receiving state "jail" tends to act like number 30 , but as a shipping state it tends to act like 10 (= visiting jail) with a delay which depends on $p$. It is mainly for this reason that the position frequencies turn out to be unequal, but the chance

cards are another strong factor. If we think of the movement around the board as a fluid flow, then 30 seems to be a sink which feeds a source at 10 .

It is easy to relate the position frequencies per throw to the position frequencies per turn. In fact, since in any sequence of throws, the corresponding number of turns is precisely the number of unprimed states in the sequence, the average number of turns per throw is the probability of being in an unprimed state. Let $f_{n}, f_{n^{\prime}}, f_{n^{\prime \prime}}$ be the state frequencies of the per throw chain. Then

$$
\alpha=\sum_{n=0}^{39} f_{n}+f_{300}+f_{301}+f_{301}
$$

is the probability of being in an unprimed state, and hence for each $n,\left(f_{n}+f_{n^{\prime}}+\right.$ $\left.f_{n^{\prime \prime}}\right) / \alpha$ is the frequency of position $n$ per turn. (For convenience, define terms with subscript 30, primed or not, to be 0 ) Thus, all the important information about the per turn chain can be obtained from the per throw chain. We shall also find how to relate the two transition matrices. (See $\S 9$ )

\section{THE PER THROW MATRIX}

We order the states in three segments of 39 each (unprimed, then single primed, the double primed) followed by the three jail states. Accordingly, we arrange the transition matrix of the per throw chain into nine $39 \times 39$ blocks with a border three entries wide. This gives the matrix the appearance

$$
\left[\begin{array}{cccc}
A & B & 0 & J_{3} \\
A & 0 & B & J_{3} \\
A & 0 & 0 & J_{4} \\
J_{1} & J_{2} & 0 & J_{5}
\end{array}\right]
$$


In naming positions in these matrices we shall always use the number of the corresponding state, not the ordinal number of the row or column.

The blocks of 0's in this matrix indicate the impossibility of passing directly between certain states. For example, it is impossible to go from a state $n, n^{\prime \prime}$, or jail to a state $m^{\prime \prime}$, or from a state $n^{\prime}$ or $n^{\prime \prime}$ to a state $m^{\prime}$. Several of the blocks are repeated, since it is equally probable to pass from the states $n, n^{\prime}$, or $n^{\prime \prime}$ to a state $m$ (block $A$ ); the probability of passing from $n$ to $m^{\prime}$ is the same as from $n^{\prime}$ to $m^{\prime \prime}$ (block $B$ ); and the probability of going to jail is the same from either $n$ or $n^{\prime}$ (block $J_{3}$ ). It is possible to reach states 301 and 302 only from 300 , so in the last two columns only block $J_{5}$ has non zero entries. These two columns of $J_{5}$ involve the parameter $p$, and in fact they are

$$
\left[\begin{array}{cc}
5(1-p) / 6 & 0 \\
0 & 5(1-p) / 6 \\
0 & 0
\end{array}\right] .
$$

In block $A$ we enter the transition probabilities which deal with non doubles throws: advance 3 with probability $1 / 18, \ldots$, advance 7 with probability $1 / 6, \ldots$, plus whatever contributions there are from position-transfer cards. In block $B$ we have the transition probabilities which deal with doubles throws: advance $2,4,6,8,10$, or 12 , each with probability $1 / 36$, again plus whatever contributions there are from position-transfer cards.

In either case, we can compute the contributions from the position-transfer cards as follows: compute the transition probabilities into the chance and community chest positions as if there were no transfer cards, getting 6 columns for each of $A$ and $B$. Divide these columns by 16 and add these $1 / 16$ shares to the columns of the positions which have the possibility of a transfer due to a card from the corresponding chance or community chest position. Leave $3 / 8$ of the chance columns and $7 / 8$ of the community chest columns in their own positions. There is one tricky item in all this, in that the "back up 3 spaces" chance card can move the pawn from Chance 36 to Community Chest 33, and then conceivably an additional transfer to the jail or to Go could occur. Thus, we must add $1 / 16$ share of column 36 to column33 before we divide column 33 into shares $1 / 16,1 / 16$, and $7 / 8$ to be disbursed to columns 0,300 , and 33 , respectively.

There are just two rows whose entries are non constant functions of $p$, namely, rows 300 and 301 in blocks $J_{1}$ and $J_{5}$. Rows 300 and 301 of $J_{1}$ are just $p A_{10}$, where $A_{10}$ is row 10 of $A$. Row 302 of $J_{1}$ equals $A_{10}$. All three rows of $J_{2}$ are the same as row 10 of $B$. The non zero column 300 of $J_{4}$ is column 300 of $J_{3}$ plus the row-sum column of $B$, since the row sums of the whole matrix are all 1 . The row sums of $B$ are almost $1 / 6$, and the addition of this $1 / 6$ to the entries of $J_{3}$ to get the entries of $J_{4}$ can be considered, essentially, as the effect of being sent to jail for throwing doubles a third time.

When we multiply this matrix by row matrices, in actual hand computations, we treat the computation algorithmically, as reflected in the structure of the matrix described above. Thus, in the first 39 row $\times$ column operations we multiply $A$ by the sum of the three 39-element segments and then add in the contribution from $J_{1}$. Also, since some of the columns of $A$ (or $B$ ) are obtained by operating on more primitive columns, (e.g., adding $1 / 16$ of primitive chance or community chest columns), we first perform the row $\times$ column operation with the primitive columns and then add to obtain the final results. These primitive columns of $A$ and $B$ 
are rational numbers with common denominators 18 or 36 , respectively, and small integers $(1,2$, or 3 ) as numerators, so the division by 18 or 36 is saved until all the other multiplications and additions are done. This procedure simplifies the computations and reduces round off error to a minimum.

\section{IRREDUCIBILITY AND NON PERIODICITY}

A finite Markov chain is said to be irreducible if every state can be reached from every other state. The two chains described above are irreducible except when $p=1$. In the case $p=1$, the states 301 and 302 can never be reached from the others, so the remaining states form an irreducible chain by themselves.

For any state $i$ of an irreducible chain, let $d_{i}$ be the greatest common divisor of the set $N_{i}$ of positive integers $n$ such that starting from $i$ it is possible to return to $i$ in $n$ steps. Then $d_{i}=d$ is the same for all states $i$. (Proof: If $i$ and $j$ are different states, then there is a path of length $r$ from $i$ to $j$, and a path of length $s$ from $j$ to $i$. Thus, $r+s \in N_{j}$, and $d_{i}, d_{j}$ both divide $r+s$. If $n \in N_{i}$, then the path of length $s$ from $j$ to $i$, followed by a loop of length $n$, followed by the path of length $r$ from $i$ to $j$, forms a loop of length $s+n+r$ based at $j$. Hence $d_{j}$ divides $s+n+r$ and therefore $d_{j}$ divides $n$.) If $d>1$, the chain is said to be periodic with period $d$. If a chain has period $d$, the set of states can be partitioned into subsets $C_{0}, C_{1}, \ldots, C_{d-1}$ such that if the state at time $k$ belongs to the set ${ }_{r}$, then the state at time $k+1$ must be in $C_{r+1}$ (identifying $C_{d}$ with $C_{0}$ ).

The chains for Monopoly are clearly not periodic, since loops of length 1 are possible at state 0 . Even if there were no position-transfer cards, the chains would not be periodic, since it is possible to pass from, say, state 0 to state 10 in one step (a throw of $6+4$ ) or two steps (throws of $3+2$ on two turns in a row).

\section{State vectors}

A state vector for a Markov chain is a row vector with one entry for each state, such that the entries are nonnegative and have sum 1 . The state vector at time $k$ is the state vector whose $j^{\text {th }}$ component is the probability of being in state $j$ at time $k$. Thus, for Monopoly, the state vector at time 0 has 1 in the Go position, 0 's elsewhere. If the state vector at time $k$ is $X$, then the state vector at time $k+n$ is the matrix product $X P^{n}$, where $P$ is the transition matrix of the chain. In particular, the element $p_{i j}^{(n)}$ in row $i$, column $j$ of $P^{n}$ is the probability, starting from state $i$, of being in state $j$ after $n$ steps. Obviously if we know how $P$ acts on all state vectors, we know $P$. We use this simple fact to compute the matrix for the per turn chain in terms of the matrix for the per throw chain.

\section{THE PER TURN MATRIX}

A state vector $\left(X, X_{J}\right)$ for the per turn chain can be augmented with 0's to get a state vector $\left(X, 0,0, X_{J}\right)$ for the per throw chain. At the next throw the state vector will be, according to $\S 6$,

$$
\left(X A+X_{J} J_{1}, X B+X_{J} J_{2}, 0, X J_{3}+X_{j} J_{5}\right) \text {. }
$$

However, if we think in terms of turns rather than throws, the components $X A+$ $X_{J} J_{1}$ and $X J_{3}+X_{J} J_{5}$ are already at the next turn, while $X B+X_{J} J_{2}$ requires at least one more throw to get it to the next turn. Applying the per throw matrix on $\left(0, X B+X_{J} J_{2}, 0,0\right)$ gives $\left(X B A+X_{J} J_{2} A, 0, X B^{2}+X_{J} J_{2} B, X B J_{3}+\right.$ 
$X_{J} J_{2} J_{3}$ ), of which the component $X B^{2}+X_{J} J_{2} B$ has not yet reached the next turn. Applying the per throw matrix to this remaining component gives $\left(X B^{2} A+\right.$ $\left.X_{J} J_{2} B A, 0,0, X B^{2} J_{4}+X_{J} J_{2} B J_{4}\right)$. Adding the non primed components of these three vectors gives the per turn state vector which follows $\left(X, X_{J}\right)$ :

$\left(X\left[I+B+B^{2}\right] A+X_{J}\left[J_{1}+J_{2} A+J_{2} B A\right], X\left[J_{3}+B J J_{3}+B^{2} J_{4}\right]+X_{J}\left[J_{5}+J_{2} J_{3}+J_{2} B J_{4}\right]\right)$.

Evidently the matrix for the per turn chain is

$$
\left[\begin{array}{cc}
{\left[I+B+B^{2}\right] A} & J_{3}+B J_{3}+B^{2} J_{4} \\
J_{1}+J_{2}[I+B] A & J_{5}+J_{2} J_{3}+J_{2} B J_{4}
\end{array}\right] .
$$

Now suppose that $\left(F, F^{\prime}, F^{\prime \prime}, F_{J}\right)$ is an eigenvector of the per throw matrix having eigenvalue 1 . Computing what this means we get

$$
\begin{gathered}
F^{\prime}=F B+F_{J} J_{2}, \\
F^{\prime \prime}=F^{\prime} B=F B^{2}+F_{J} J_{2} B, \\
F=\left(F+F^{\prime}+F^{\prime \prime}\right) A+F_{J} J_{1}=F\left(I+B+B^{2}\right) A+F_{j}\left(J_{1}+J_{2} A+J_{2} B A\right), \\
F_{J}=\left(F+F^{\prime}\right) J_{3}+F^{\prime \prime} J_{4}+F_{J} J-5 \\
=F\left(J_{3}+B J_{3}+B^{2} J_{4}\right)+F_{J}\left(J_{5}+J_{2} J_{3}+J_{2} B J_{4}\right) .
\end{gathered}
$$

The last two of these equations say that $\left(F, F_{J}\right)$ is an eigenvector of the per turn matrix having eigenvalue 1 . We shall see that this amounts to a proof of the relation between position frequencies found (less convincingly) at the end of $\S 5$.

\section{THE BASIC THEORY}

The following facts about an irreducible, finite, non periodic Markov chain with matrix $P$ are proved in $[\mathrm{F}]$.

(1) 1 is an eigenvalue of $P$ and the corresponding row eigenspace is onedimensional. This row eigenspace is spanned by a unique state vector $F=\left(f_{i}\right)$ having all entries positive. It is the unique solution of the system of equations

$$
\sum_{i} f_{i} p_{i j}=f_{j}
$$

for all $j$ (in matrix form: $F P=F$ ),

$$
\sum_{i}=1 \text {. }
$$

(2) All other eigenvalues of $P$ have magnitude less than 1.

(3) For any state vector $X, \lim _{n \rightarrow \infty} X P^{n}=F$. In particular $\lim P^{n}$ is the square matrix having all rows equal to $F$.

(4) If the system begins in any state, and $R_{i}(n)$ is the number of times state $i$ occurs in the first $n$ steps, then

$$
\lim _{n \rightarrow \infty} \frac{R_{i}(n)}{n}=f_{i}
$$

with probability 1.

(5) If the system begins in any state and $n_{0}(i), n_{1}(i), \ldots, n_{k}(i), \cdots$ are the steps, in increasing order, for which the state is $i$, then

$$
\lim _{k \rightarrow \infty} \frac{n_{k}(i)-n_{0}(k)}{k}=\frac{1}{f_{i}}
$$

with probability 1 . 
The entries $f_{i}$ of $F$ are called the limit frequencies; their reciprocals $\mu_{i}=1 / f_{i}$ are called the mean recurrence times.

The column $E$ having all entries 1 is a basis of the column eigenspace with eigenvalue 1 . It follows that the space of row vectors $E^{\text {perp }}$, those with sum of entries equal to 0 , is invariant under $P$, complementary to the span of $F$, and that all other row eigenvectors are in $E^{\text {perp }}$. The other eigenvalues of $P$ may be (and some are in the case of the Monopoly chains) complex, not real, so we either must allow complex entries or deal with invariant two-dimensional subspaces of $E^{\text {perp }}$ as a real vector space. Moreover, it follows from (1) that the restriction of $P-I$ to $E^{\text {perp }}$ is nonsingular, and from (3), for any $X \in E^{\text {perp }}$ the infinite sum $\sum X P^{n}$ exists and converges to $X(I-P)^{-1}$.

\section{Computational techniques}

The system of 121 equations in 120 unknowns $f_{i}$, given above under (1) in the case of the per throw chain, is consistent and has a unique solution. However, if we try to solve by discarding one of the equations and trying some elimination procedure, the task is quite formidable. Also, as anyone having modest experience knows, round-off error in solving large linear systems can be quite severe. Hence, it was decided to use the limit formula in (3) as the computational tool for finding $F$.

Setting $p=1 / 2$ and choosing a likely looking initial approximation $X_{0}$ for $F$, we let $X_{n}=X_{0} P^{n}$ and $Y_{n}=X_{n}-X_{n-1}=Y_{n-1} P$. Thus, $X_{n}=X_{0}+Y_{1}+\cdots+$ $Y_{n}$. It was found that $Y_{n}$ was 0 to within 3 significant figures (each entry having magnitude $<.00005$ ) for $n$ about 30 . The reason for using the difference $Y_{n}$ was to obtain entries of smaller magnitude, thus facilitating the computation. the sum $X_{0}+Y_{1}+\cdots+Y_{n}=X_{0}^{\prime}$ was then operated on by $P$ as a check, and errors which appeared were again eliminated by iteration of $P$ on $Y_{1}^{\prime}=X_{0}^{\prime} P-X_{0}^{\prime}$.

These computations employed an abacus as an adding machine, using the algorithm described at the end of $\S 6$. It would have been easier, of course, to use a computer, but the remarkable thing is that iterative computations are at all feasible with such simple equipment dealing with such a large matrix. The natural grouping into blocks of 39 entries helped immensely.

The results are given in table I for three values of $p$. they are normalized so that the entries represent frequency per turn. The first two columns are of the form $\left(f_{n}+f_{n^{\prime}}+f_{n^{\prime \prime}}\right) / \alpha$, as they should be according to the discussion of $\S 5$, where the $f_{n}, f_{n^{\prime}}, f_{n^{\prime \prime}}$ are the frequencies of the states in the perthrow chain and $\alpha$ is the sum of the unprimed frequencies. The jail is listed under number 30 and is not broken down into its three state, only their total. In the last three columns we have listed the break-down into the frequencies of the states in the per throw chain for the single case $p=1 / 2$. They are normalized so that the sum of the unprimed frequencies is 1 . The case $p=1 / 2$ was the only one done directly by the method described above; for a description of the method used to find the frequencies as functions of $p$, see $\S 12$. 
TABLE I. Position Frequencies.

\begin{tabular}{|c|c|c|c|c|c|c|}
\hline \multirow[t]{2}{*}{$n$} & \multirow[t]{2}{*}{ osition } & \multirow{2}{*}{$\begin{array}{c}\text { Remain in J } \\
\quad p=0\end{array}$} & \multirow{2}{*}{$\begin{array}{l}\text { Leave } \mathrm{J} \\
\qquad p=1\end{array}$} & \multicolumn{3}{|c|}{$\begin{array}{l}\text { Normalized state } \\
\text { frequencies, } p=.5\end{array}$} \\
\hline & & & & $n$ & $n^{\prime}$ & $n^{\prime \prime}$ \\
\hline 0 & Go & .0345904 & .0368 & .0306 & .0046 & .0007 \\
\hline 1 & Mediterranean & .0238252 & .0252 & .0200 & .0039 & .0007 \\
\hline 2 & Comm & .0210683 & .0223 & .0187 & .0027 & .0004 \\
\hline 3 & Baltic & .0241763 & .0256 & .0205 & .0038 & .0007 \\
\hline 4 & Income & .0260343 & .0277 & .0226 & .0039 & .0006 \\
\hline 5 & Reading RR & .0332449 & .0352 & .0285 & .0050 & .0009 \\
\hline 6 & Oriental & .0253014 & .0268 & .0222 & .0035 & .0005 \\
\hline 7 & Chance & .0096756 & .0102 & .0083 & .0014 & .0003 \\
\hline 8 & Verm & .0259620 & .0274 & .0229 & .0034 & .0005 \\
\hline 9 & Conr & .0257326 & .0272 & .0226 & .0034 & .0006 \\
\hline 10 & Visit & & .0269 & .0219 & .0038 & .0006 \\
\hline 11 & St. Charl & .0303 & .0321 & .0263 & .0043 & .0008 \\
\hline 12 & Electric & .0310 & & .0239 & .0064 & .0007 \\
\hline 13 & States & .025 & .0281 & .0231 & .0035 & .0006 \\
\hline 14 & Virg & .0287890 & .0293 & .0227 & .0057 & .0007 \\
\hline 15 & Penn & .0312800 & & .0285 & .0041 & .0007 \\
\hline 16 & St. J & .0318117 & .0 & .0261 & .0058 & .0007 \\
\hline 17 & Comn & .0272474 & .0307 & .0255 & .0033 & .0005 \\
\hline 18 & Tenr & .0334833 & .0349 & .0276 & .0059 & .0008 \\
\hline 19 & New & 34 & .0366 & .0306 & .0041 & .0006 \\
\hline 20 & Free & .0335 & .0343 & .0270 & .0061 & .0009 \\
\hline 21 & Kent & & & .0276 & .0044 & .0007 \\
\hline 22 & Chas & & & .0098 & .0023 & .0004 \\
\hline 23 & & & & .0265 & .0045 & .0007 \\
\hline 24 & Illin & & & 0312 & .0047 & .0010 \\
\hline 25 & & & & .0295 & .0053 & .0008 \\
\hline 26 & Atla & & & .0265 & .0040 & .0008 \\
\hline 27 & & & & .0256 & .0047 & .0007 \\
\hline 28 & Wate & & & .0272 & .0045 & .0009 \\
\hline 29 & Marv & & & .0247 & .0046 & .0007 \\
\hline 30 & Jail t & & & .0459 & .0191 & .0080 \\
\hline 31 & Pacific & & & .0257 & .0046 & .0008 \\
\hline 32 & North C & & & .0265 & .0034 & .0006 \\
\hline 33 & Com & & & .0226 & .0041 & .0007 \\
\hline 34 & Penı & & & .0251 & .0034 & .0005 \\
\hline 35 & ine $R R$ & & .0289 & .0231 & .0043 & .0008 \\
\hline 36 & Chan & .0096825 & .0102 & .0084 & .0014 & .0002 \\
\hline 37 & Par & .0244 & .0260 & .0204 & .0042 & .0008 \\
\hline 38 & Luxu & .0243572 & .0260 & .0217 & .0032 & .0005 \\
\hline 39 & Boardwalk & .0294734 & .0312 & .0248 & .0048 & .0009 \\
\hline
\end{tabular}




\section{The PARAMETER $p$}

Above we indicated how the limit frequencies, $F(1 / 2)$, were computed. To determine the nature of $F(p)$, consider the system of equations in $(1), \S 10$. One of these equations is dependent and may be discarded, leaving 120 equations in 120 unknowns which may be written in matrix form $F(p) C(p)=D$, where $C(p)$ is square and nonsingular. Since $p$ appears linearly and in only two rows of $C(p)$, it follows from Cramer's rule that $F(p)$ is a rational function of $p$ having quadratic numerator and denominator. In particular, $F$ is an analytic function of $p$. It will be convenient in the following analysis to translate the variable to the point $p=1 / 2$, so we let $t=p-1 / 2$ and $F_{0}=F(1 / 2)$. The derivative of $P(t)$ is constant and we shall denote it by $Q=P^{\prime}(t)$. Note that the row sums of $Q$ are 0 . Let $P_{0}=P(1 / 2)$, so $P=P_{0}+t Q$.

We shall determine $F$ by considering its power series in powers of $t$ and using matrix manipulations to condense these series into the desired rational quadratic. By the second equation of (1) the $n^{\text {th }}$ derivative $F^{(n)}$ has sum of entries 0 for $n>0$, so we shall be working mostly in the space $E^{\text {perp }}$. Now we start with the other equation $F P=F$ and differentiate repeatedly:

$$
F^{\prime} P+F Q=F^{\prime}
$$

that is,

$$
\text { (6) } F^{\prime}(I-P)=P Q \text {. }
$$

We claim that

$$
\text { (7) } F^{(n)}(I-P)=n F^{(n-1)} Q .
$$

In fact, we have the initial step (6) of an inductive proof, and differentiating (7) again gives the recursive step:

$$
F^{(n+1)}(I-P)+F^{(n)}(-Q)=n F^{(n)} Q
$$

Besides being constant, $Q$ consists mostly of 0's. Only the rows tagged 300 and 301 in $P$ contain $p$, so only $Q_{300}$ and $Q_{301}$ are non zero. Thus, for any row vector $X$ having entries $X_{i}$,

$$
X Q=X_{300} Q_{300}+X_{301} Q_{301} .
$$

Let $S$ be the inverse of the restriction of $I-P_{0}$ to $E^{p e r p}, V=Q_{300} S, W=Q_{301} S$. Denoting the entries of 0 by $f_{i}$, we have from (6)

$$
\text { (8) } F^{\prime}(1 / 2)=F_{0} Q S=f_{300} V+f_{301} W \text {. }
$$

We claim that

$$
F^{(n)}(1 / 2)=n ! \cdot\left(f_{300}, f_{301}\right)\left[\begin{array}{cc}
V_{300} & V_{301} \\
W_{300} & W_{301}
\end{array}\right]^{n-1}\left[\begin{array}{c}
V \\
W
\end{array}\right]
$$

This follows immediately from (8) and (7) by induction. Since $F$ is analytic, we have in some interval of convergence about $t=0$,

$$
\begin{aligned}
F & =F_{0}+\sum_{n=1}^{\infty}\left(f_{300}, f_{301}\right)\left[\begin{array}{cc}
V_{300} & V_{301} \\
W_{300} & W_{301}
\end{array}\right]^{n-1}\left[\begin{array}{c}
V \\
W
\end{array}\right] t^{n} \\
& =F_{0}+t\left(f_{300}, f_{301}\right)\left[\begin{array}{cc}
1-V_{300} t & -V_{301} t \\
-W_{300} t & 1-W_{301} t
\end{array}\right]^{-1}\left[\begin{array}{c}
V \\
W
\end{array}\right] .
\end{aligned}
$$


We have used the fact that for a matrix $M$ close to zero,

$$
(I-M)^{-1}=\sum_{n=0}^{\infty} M^{n} .
$$

Thus, to evaluate $F$ as a function of $t$ it suffices to find

$$
V=Q_{300} S=\sum_{n=0}^{\infty} Q_{300} P_{0}^{n}
$$

and

$$
W=\sum_{n=0}^{\infty} Q_{301} P_{0}^{n}
$$

and insert them into formula (10).

The computation of $V$ and $W$ was done by iteration, as indicated. To speed up the convergence, and to gain insight into the structure of $P_{0}$, it was decided to find the larger eigenvalues of $P_{0}$. The procedures for finding these eigenvalues and for using them in the iteration is described in the next section. After the computation of $V$ and $W$ was completed, the formula for $F$ was found to be approximately

$$
\text { (11) } F=F_{0}+\frac{t}{1-.0454 t+.0249 t^{2}}\left(F_{1}+t Y\right) \text {, }
$$

where $F_{1}=F^{\prime}(1 / 2)$ and $Y$ is an appropriate linear combination of $V$ and $W$. This formula was used to compute $F(0)$ and $F(1)$ in table I. Moreover, every entry of $F$ turned out to be monotonic for $0 \leq p \leq 1$, so that for any $p$, the entries of $F(p)$ lie between those of $F(0)$ and $F(1)$.

\section{OTHER EIGENVALUes}

We cannot guarantee that $P=P_{0}$ is diagonalizable or has distinct eigenvalues with one-dimensional eigenspaces. However, the matrices which are not of this type form a set of Lebesgue measure 0 in the space of matrices, so we probably shall not go wrong if we assume $P$ is diagonalizable. Moreover, it is likely that such a bad condition in the matrix $P$ would show up in the procedure we are about to describe, but there was not the merest hint of multiplicity in eigenvalues or even bunching together among the larger eigenvalues. Later we did some Fortran matrix program calculations and determined all the eigenvalues, finding them to be distinct.

On the other hand, it is certain that $P$ has complex, non real, eigenvalues. Since $P$ is real, the complex eigenvalues must occur in conjugate pairs $\mu$ and $\bar{\mu}$. The eigenspaces belong to such a pair will also be complex conjugates, with real and imaginary parts spanning a two-dimensional real space, invariant under $P$ and annihilated by the real operator $(P-\mu)(P-\bar{\mu})=P^{2}-(\mu+\bar{\mu}) P+\mu \bar{\mu} I$. If a real row-vector $X$ is in this sum of conjugate eigenspaces, $X P^{2}, X P$, and $X$ are linearly dependent, but $X P$ and $X$ are not, since $\mu$ is not real. Conversely, if we find a vector $X$ for which $X P^{2}, X P$, and $X$ are linearly dependent, or nearly so, but $X P$ and $X$ are not, then $X$ is in a sum of conjugate eigenspaces, or close to it. If $X P^{2}+a X P+b X$ is a linear combination which is close to zero compared with $X$, then $P^{2}+a P+b I$ will nearly annihilate a sum of conjugate eigenspace whose eigenvalues almost satisfy $\mu^{2}+a \mu+b=0$.

If $\mu$ is larger in magnitude than the other eigenvalues of the restriction of $P$ to $E^{p e r p}$, then for any vector $X$, the component of $X$ in the $\mu-\bar{\mu}$ eigenspace dies more 
slowly than the components in the other eigenspaces when $P$ is applied repeatedly to $X$. Thus, for some $n$ we should expect that $X P^{n+2}, X P^{n+1}, X P^{n}$ are nearly linearly dependent, and we should come close to determining $\mu$ by finding a good linear approximation $-a X P^{n+1}-b X P^{n}$ for $X P^{n+2}$. After the largest eigenvalue pair has been found, we can work within the rage space of the quadratic annihilator of the first to find the second largest by the same technique.

This technique is similar to the technique for finding the principal modes of small vibrations of a mechanical system about a point of stable equilibrium. However, in a mechanical system the matrix is symmetric, so the eigenvalues are real.

The largest eigenvalues of $P=P_{0}$ turned out to have magnitudes of approximately .85 and .69 , with quadratic annihilators $P^{2}-.73 P+.72 I$ and $P^{2}+.82 P+.47 I$. (These were for the per throw matrix. The later calculations for the per turn matrix on a mainframe gave $P^{2}-.492 P+.625 I$ and $P^{2}+.802 P+.333 I$.) In the first trials, the sizable component for the second of these interfered with the accurate determination of the first. However, after a crude approximation to the second one was found, it was used to reduce the relative size of the second one's component, as compared with the first one's, in a certain trial vector. This produced a better trial vector for the determination of the first one.

By multiplying the first two quadratics together (for the per turn matrix) and normalizing so that the sum of coefficients is 1 , we get a quartic in $P$ which nearly annihilates the 4-dimensional space of the two largest eigenvalue pairs: $M=.0861 I+.1393 P+.2330 P^{2}+.1280 P^{3}+.4136 P^{4}$. This normalization is convenient for the purpose of evaluating sums $\sum X P^{n}$, since we have

$$
\begin{array}{rl}
\sum X P^{n} & =\sum X M P^{n}+(1-.0861) X+(1-.0861-.1393) X P \\
& +(1-.0861-.1393-.2330) X P^{2} \\
& +(1-.0861-.1393-.2330-.1280) X P^{3} \\
=\sum X & M P^{n}+.9139 X+.7746 X P+.5416 X P^{2}+.4136 X P^{3}
\end{array}
$$

Since the larger components in $X M$ belong to eigenvalues of even smaller magnitude, the terms $X M P^{n}$ die off very rapidly as we increase $n$. The computational advantage from finding the larger eigenvalues derives from this fact.

When we let $X=Q_{300}$ or $Q_{301}$, then only six terms of $\sum X M P^{n}$ are required. (The later calculations of the next eigenvalue, namely, -.3620 show that all the other components will have decreased to at most about .006 times their initial magnitude.)

The magnitudes of these major eigenvalues indicate the rapidity of convergence to the limit frequencies. For example, multiplying a state vector by $P^{20}$ reduces the size of the projection to $E^{\text {perp }}$ by a factor of $(.7905)^{2} 0=.009$ or better. For a pure state vector, corresponding to being on some one position, the sizes of these components in $E^{\text {perp }}$ would seem to be about the same as that of the limit frequency vector. Thus, starting at any position, the probability of being on any other position after 20 turns is within one percent of the limit frequency of that other position.

Our evidence for thinking that the sizes of the components in $E^{\text {perp }}$ of pure state vectors are about the same as the limit frequencies is based on an analysis of another Markov chain. We can view the per turn chain as a perturbation of a random walk on 40 positions arranged in a cycle, where advance from one position to the next is determined by throwing dice. An extra throw is given for a first or 
second doubles throw. Since the matrix for this chain is cyclic, the eigenvectors are of the form $\left(1, \alpha, \alpha^{2}, \ldots, \alpha^{39}\right)$, where $\alpha$ is any $40^{t h}$ root of 1 . Thus, all the eigenvalues and components of pure state vectors can be readily computed. These agree well with the data we have on the Monopoly chain, and hence support the suppositions made above.

\section{EXPECTED BANK EXCHANGES}

The limit frequencies can be used to obtain the expected values of the exchanges of currency. Here we examine those which occur between a player and the bank. We shall not attempt to estimate certain expenses which have a more irregular pattern: original cost of property and buildings, cost of mortgaging property, etc. However, by comparing the expected loss in rent on one property to the gain from another, we could resolve the problem of whether it is wise to mortgage the one in order to finance building on the other.

Each player has a probability of gaining in regular exchanges with the bank. The principal source of gain is the salary of $\$ 200$ for each time Go is passed. This requires that we compute the frequency of passing Go per turn. The entries $g_{n}$ in columns 1, 2 in table I give the probability of being on a given position during any turn, including the possibility of being there on a second or third throw of the turn. The probability of moving past Go by advancing the number of steps indicated by the dice for that throw is

$$
\begin{aligned}
A=\frac{1}{36}\left(g_{28}\right. & +3 g_{29}+10 g_{31}+15 g_{32}+21 g_{33}+26 g_{34} \\
& \left.+30 g_{35}+33 g_{36}+35 g_{37}+36 g_{38}+36 g_{39}\right) .
\end{aligned}
$$

The other means of passing Go is by a position-transfer card. The probability of obtaining one of these is determined by the frequency of the Chance and Community Chest positions. Thus, the probability of lighting on Chance 7 during a turn is $(8 / 3) g_{7}$. From Chance 7 there are two of 16 chance card which will transfer position onto or past Go. (Advance to Go, take a ride on the Reading RR.) The contribution to passing Go from Chance 7 is thus $(1 / 3) g_{7}$. This includes the possibility of passing Go twice in a turn. The total contribution to passing Go in this way is

$$
B=\frac{1}{3} g_{7}+\frac{1}{2} g_{22}+\frac{7}{6} g_{36}+\frac{1}{14}\left(g_{2}+g_{17}+g_{33}\right) .
$$

hence the expected salary from Go per turn is $\$ 200 \cdot(A+B)$, which amounts to about $\$ 40$.

The other sources of regular exchange with the bank are income and luxury taxes (a loss), Community Chest and Chance card (a net gain), and the cost of getting out of jail Another loss comes from the card "Assessment of Property(Community Chest)" and "General Repairs (Chance)" and depends on the number of houses and hotels owned, as well as the frequency of the Community Chest and Chance positions. The expected values of these exchanges are listed in table II. The Assessment and General Repairs are listed last without inclusion in the total.

The value of a "Get out of jail free" card was considered to be $\$ 50$ dollars, since it is always possible to wait until it can be used to save payment of the fee for getting out of jail.

In computing the cost of leaving jail we presume that the decision is made whether or not to leave before the dice are thrown, even when $p$ is not 0 or 1 . Thus, 
the expected value is

$$
\$ 50\left(p g_{300}+p g_{301}+\frac{5}{6} g_{302}\right) .
$$

(With probability $1 / 6$ a "free ride" will be obtained by throwing doubles on the third turn.)

TABLE II

Expected income from bank, $\$$ per turn

\begin{tabular}{|c|c|c|}
\hline & $p=0$ & $p=1$ \\
\hline Salary (pass Go) & 39.92300 & 41.16 \\
\hline Income \& Luxury taxes & -7.03365 & -7.49 \\
\hline Community Chest & 1.86887 & 2.03 \\
\hline Chance & 1.24390 & 1.29 \\
\hline Get out of jail fee & -1.28487 & -2.35 \\
\hline Total & 33.71725 & 34.64 \\
\hline \multicolumn{3}{|l|}{ Assessment and } \\
\hline -each house & -.34592 & -.37 \\
\hline - each hotel & -.14338 & -1.22 \\
\hline
\end{tabular}

TABLE III

Expected property-group income, with hotels, \$'s per opponents' turn

\begin{tabular}{lrr}
\hline & $p=0$ & $p=1$ \\
\hline 1,3 & 16.84 & 17.8 \\
$6,8,9$ & 43.65 & 46.1 \\
$11,13,14$ & 68.02 & 71.5 \\
$16,18,19$ & 95.40 & 101.2 \\
$21,23,24$ & 103.65 & 110.9 \\
$26,27,29$ & 103.74 & 110.3 \\
$31,32,34$ & 114.70 & 121.9 \\
37,39 & 95.62 & 101.4 \\
Railroads (1 owner) & 27.33 & 29.2 \\
Utilities (1 owner) & 4.37 & 4.5
\end{tabular}

\section{EXPECTED RENT}

The expected rent per opponents' turn can be computed for each property owned, at any stage of development (i.e., number of houses and hotels). We list this rent for each property group, at the greatest stage of development, in table III. To determine the maximum rent-producing capacity of a group, the corresponding figure should be multiplied by the number of opponents, and then reduced by the building costs determined from the last lines of table II. Thus, from group 11, 13, 14 (St. Charles, etc.) with hotels against two opponents one should anticipate an income of $2 \times 68.016-3 \times 1.14338=\$ 132.602$ per turn (taking $p=0$ ).

The difference in income when operating with $p=0$ and $p=1$ is $\$ 1.27$ per turn, from table II. The only property groups for which the difference in rents in these tow modes is less than 1.27 are 1,3 (difference $=1.0$ ) and utilities $(0.1)$, from table III. If an opponent has any other group, fully developed, then it is better to operate with $p=0$ : to operate instead at $p=1$ costs more in additional rent to that opponent than one gains in additional income from the bank. This verifies that the strategy of staying in jail as long as possible is generally best. Of course, the point at which this strategy becomes preferred depends on the stage of development of the opponents' properties. Perhaps a good rule of thumb would be to switch whenever all opponents' total rent is half as much as 6, 8, 9 fully developed, for which the difference is 2.5 .

The difference in frequency within a group indicates that there is an optimum order of placing houses purchased on the two or three properties. In most cases it is better to build first on the most expensive of the group. In the occasional exception the advantage is pretty slight, no more than $\$ .50$ per opponents' turn. The major example of this is in the group 31,32, 34, where the first one to get four houses should be 31 (Pacific), and not the obvious-looking choice of 34 (Pennsylvania). Generally, when the increment in rent for all three in a group is the same for a given house, the property with the higher frequency should get the house. Usually 
the more expensive property in the group has a larger rent increment, which more than offsets frequency considerations.

As a minimum guide to values when buying and selling property, table III would serve well. If the possibility of property transactions is remote, table III could be used to determine who stands to gain the most over a long period of play (for a given property distribution), and hence who should be considered the winner.

Postscript. The above version of the detailed description of modeling Monopoly after a Markov chain was retyped in November, 1991, with very few changes in the text from the 1969 version, and again in June, 2003, with a number minor corrections. A summary version was published in Mathematics Magazine in 1972. The tables in the new version given here have been revised to correct some errors that appeared in both the original and in the 1972 summary; more accurate figures have been provided for the case $p=0$. This more accurate data was obtained by using a Fortran program.

In 1978 one author (RLB) wrote a program to obtain accurate eigenvalues and eigenvectors for the per turn matrix (besides eigenvalue 1). The five largest of these turned out to be two complex conjugate pairs and a real one:

$$
\begin{gathered}
0.2460886 \pm 0.7511934 i \text { with magnitude } 0.7904752 \\
-0.4008098 \pm 0.4153999 i \text { with magnitude } 0.5772397 \\
-0.3619940
\end{gathered}
$$

The remaining eigenvalues are all of magnitude less than 0.275 .

In the intervening years there have been independent analyses of the frequencies, conceived differently, but producing exactly the same probabilistic results. We feel that the Markov chain model has the advantage of giving rich information on the speed at which the limit frequencies are approached ("with probability 1"). However, one other version received much wider circulation $([\mathrm{H}])$ and it and a subsequent book $[\mathrm{B}]$ have interesting discussion of strategy going beyond what one can get from a knowledge of limit frequencies. The data on frequencies in [B] was taken from $[\mathrm{H}]$.

\section{REFERENCES}

[A] R. B. Ash, Basic Probability Theory, Wiley, New York, 1970, Chapter 7.

[B] Maxine Brady, The Monopoly Book, D. McKay, NY, 1974.

[F] W. Feller, An Introduction to Probability Theory and Its Applications, vol. 1, Wiley, New York, 1950.

[H] Irving R. Hentzel, How to Win at Monopoly, Saturday Review of the Sciences, April, 1973, 44-48.

UNIVERSity OF ILLINOIS AT URBANA-ChAMPAigN

UNIVERSITY OF IlLinOIS AT URBANA-ChAMPAigN

E-mail address: bishop@math.uiuc.edu 